\title{
A rare presentation of zygomycosis (mucormycosis) and review of the literature
}

\author{
M Karanth, P Taniere, J Barraclough, J A Murray
}

J Clin Pathol 2005;58:879-881. doi: 10.1136/icp.2004.021816

Zygomycosis (mucormycosis) is a rare fungal infection seen most often in association with prolonged neutropenia. Intestinal zygomycosis is extremely rare and difficult to diagnose, but it is important not to miss, because early medical and surgical treatment can improve survival. This report describes a 56 year old woman who developed this infection while receiving chemotherapy for acute lymphoblastic leukaemia. Medical and surgical measures proved unsuccessful because there was a delay in diagnosis and institution of appropriate treatment.

\section{Z} ygomycosis is a rare invasive fungal infection seen most often in patients with haematological malignancies, particularly in the neutropenic phase. ${ }^{1}$ It is the third most common cause of fungal infection after candidiasis and aspergillosis. ${ }^{1-3}$ Intestinal zygomycosis is extremely rare $^{145}$ and it is difficult to obtain a histological diagnosis, although it may be possible by means of computerised tomography guided biopsy of the abnormal mucosa. ${ }^{6}$ The diagnosis of zygomycosis is rarely suspected and antemortem diagnosis is made in only $23-50 \%$ of cases. ${ }^{7}$ Early medical and surgical treatment could prevent further dissemination. ${ }^{5}$ Zygomycosis has a high mortality of $70-100 \%,{ }^{15}$ but some patients may be cured by surgical excision and amphotericin. ${ }^{15}$

"The diagnosis of zygomycosis is rarely suspected and antemortem diagnosis is made in only $23-50 \%$ of cases"

We report a patient with disseminated intestinal zygomycosis who presented in the neutropenic phase, post chemotherapy for acute lymphoblastic leukaemia.

\section{CASE REPORT}

We report a 56 year old woman who presented with bone marrow relapse of acute lymphoblastic leukaemia (Philadelphia negative). She was started on induction chemotherapy according to the UKALL Xll protocol. She developed right iliac fossa pain and tenderness in the neutropenic phase (absolute neutrophil count, $0.0 \times 10^{9}$ / litre). While she was on steroids, an ultrasound scan of the abdomen showed an inflamed ascending colon and ileum. The differential diagnoses considered were post chemotherapy typhlitis and appendicitis. In view of severe neutropenia she was initially managed conservatively, but ultimately required right hemicolectomy and ileostomy when she developed peritonitis. She developed neutropenic sepsis and was treated with appropriate antibiotics and the lipid form of amphotericin.

Histology of the terminal ileum showed normal submucosa and muscularis propria. The subserosa showed moderate mixed inflammatory infiltration and numerous granulomas.
One section showed fungal hyphae in a large submucosal vessel on haematoxylin and eosin staining, with features suggestive of zygomycosis-numerous hyphae in the necrotic intestine. The hyphae were wide, non-septate, branched at right angles, and looked empty on haematoxylin and eosin staining. Although other infections such as rhizopus or absidia could not be excluded absolutely without culture, the typical histological features strongly suggested zygomycosis.

She was started on a lipid form of amphotericin but unfortunately developed serious bleeding from the abdominal wound and stoma. Bleeding is a known complication of this infection, particularly when it invades the vessel wall as shown in fig l. Computerised tomography of the abdomen showed features suggestive of ischaemic bowel, confirmed on laparotomy, which showed multiple abscesses in the peritoneal cavity and faecal peritonitis. Additional histology of the ileum and jejunum showed florid fungal hyphae, indicating that this patient developed disseminated infection and died as a result of intestinal zygomycosis.

\section{DISCUSSION}

We present a case of intestinal zygomycosis, which is an extremely rare site for this infection. Zygomycosis is a rare invasive fungal infection, which is most often seen in immunosuppressed patients, particularly during prolonged neutropenia. It is caused by various members of the phycomycetes, such as rhizopus, mucor, and absidia. A retrospective study by Pagano et al identified 59 patients with haematological malignancy and mucormycosis. ${ }^{1}$ The most common form of presentation was pulmonary mucor, which accounted for $64 \%$ and involved orbital and maxillofacial areas in $24 \%$. They described only one patient with intestinal

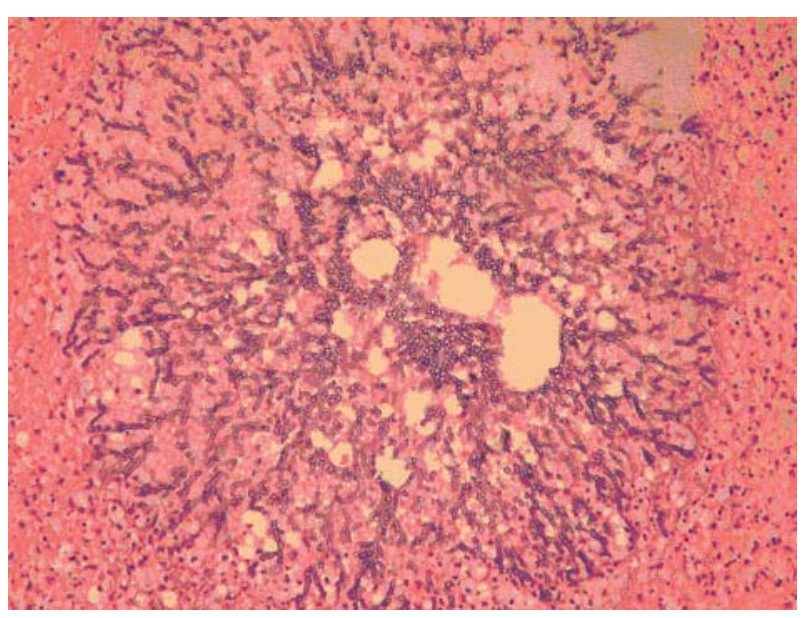

Figure 1 A subserosal vessel filled with numerous wide non-septate and empty looking hyphae characteristic of zygomycosis. Haematoxylin and eosin stain; original magnification, $\times \mathbf{4 0 0}$ magnification. 
zygomycosis. This infection carries a very high mortality rate of $79-100 \% .^{145}$

Gastrointestinal zygomycosis has been reported in neonates. It presents as necrotising enterocolitis and is invariably fatal. $^{8}{ }^{9}$

Ter Borg et al reported a fatal case of mucormycosis presenting as an appendiceal mass with metastatic spread to the liver during the neutropenic phase in a patient with $\mathrm{T}$ lymphoblastic leukaemia. ${ }^{9}$ The diagnosis was made by needle aspiration from the liver and fungal culture. They concluded that a high index of suspicion and a maximum (invasive) diagnostic effort was needed to treat this condition.

Petrikkos et al published their 10 year experience of mucormycosis at their tertiary care centre. ${ }^{10}$ They reported 24 patients and there were no gastrointestinal zygomycoses in their series. Seven of their 24 patients did not undergo surgery and all died. They concluded that the degree of immunosuppression and prompt surgical treatment are the two most important factors contributing to good outcome.

Thomson et al reported 20 patients with gastrointestinal mucormycosis in a five year study period (1984-8). ${ }^{11}$ The stomach was the most commonly involved organ and the disease presented as peptic ulceration. They concluded that when there was histological evidence of vascular invasion by the fungus, the outcome was usually fatal, irrespective of the gut site.

\section{"A high degree of clinical suspicion is needed to diagnose this condition, particularly when it involves the intestine, because delay in diagnosis results in treatment failure"}

In the neutropenic phase, inflammation of the ileum is often caused by typhlitis post chemotherapy. However, this condition resolves spontaneously when the neutrophil count recovers. Neutropenic fever can be a common presenting feature of zygomycosis. A high degree of clinical suspicion is needed to diagnose this condition, particularly when it involves the intestine, because delay in diagnosis results in treatment failure. Patients may present with non-specific symptoms such as fever, abdominal pain, and diarrhoea. Persistent severe abdominal pain in a patient with neutropenia should alert the clinician to the possibility of a fungal infection such as zygomycosis. Histological diagnosis can be attempted using computerised tomography guided biopsy of the involved area. ${ }^{6}$ In suspected cases, extensive and aggressive diagnostic and therapeutic procedures are essential to improve the outcome in these patients. ${ }^{3}$ Histology characteristically shows hyphae that are wide, non-septate, branch at right angles, and look empty when haematoxylin and eosin stained. There is no readily available polymerase chain reaction assay for mucormycosis.

The treatment of choice is amphotericin, preferably the lipid formulation, ${ }^{1}$ because adequate dosage can be delivered with reduced renal toxicity. The common prophylactic antifungal agents such as fluconazole and itraconazole are not active against this fungus. ${ }^{13}$ Newer antifungal agents such as voriconazole and caspofungin also showed no activity against mucor in clinical and in vitro studies. ${ }^{12-14}$

The new triazole antifungal agent, posaconazole, has been compared with amphotericin B, voriconazole, and itraconazole in in vitro studies. Posaconazole was significantly more active than voriconazole and other agents. ${ }^{15}$ The in vivo activity of posaconazole against mucor species has also been tested on an immunosuppressed mouse model. ${ }^{16}$ This drug could have potential for clinical development in the treatment of zygomycosis. ${ }^{16}$

Surgical resection of the involved tissue along with amphotericin improves survival. ${ }^{13}{ }^{17}$ However, the surgical
Take home messages

- We report a rare case of intestinal zygomycosis that failed to respond to combined surgical and medical treatment

- We think that the fatal outcome was partly the result of the delay in diagnosis and treatment

- Medical treatment alone is invariably unsuccessful in gastrointestinal zygomycosis, and combined antifungal treatment and surgical removal of the involved area is essential

option may not be feasible because of involvement at multiple sites, low platelet and neutrophil counts, and poor performance status. The outcome in these individuals is less favourable. Studies have shown that without surgical resection, this infection is inevitably fatal. Therefore, it is very important to consider the surgical approach. ${ }^{9}{ }^{10}$ Antifungal treatments alone are insufficient to treat this condition.

In summary, we report an extremely rare form of zygomycosis that failed to respond to combined surgical and medical treatment; we believe that this was partly a result of the delay in diagnosis and treatment. It cannot be overemphasised that medical treatment alone in gastrointestinal zygomycosis is invariably unsuccessful and combined antifungal treatment and surgical removal of the involved area is paramount to success.

\section{Authors' affiliations}

M Karanth, J A Murray, Department of Haematology, University Hospital of Birmingham, Birmingham B15 2TH, UK

P Taniere, Department of Histopathology, University of Birmingham J Barraclough, Department of Surgery, University Hospital of Birmingham

Full consent was given for the publication of this case report.

Correspondence to: Dr J A Murray, University Hospital Birmingham NHS Trust, Edgbaston, Birmingham, B15 2TH, UK; Jim.murray@uhb. nhs.uk

Accepted for publication 1 November 2004

\section{REFERENCES}

1 Pagano L, Offidani M, Fianchi L, et al. Mucormycosis in hematologic patients. Haematologica 2004;89:207-14.

2 Eucker J, Sezer O, Graf B, et al. Mucormycosis. Mycoses 2001;44:253-60.

3 Pagano L, Ricci P, Tonso A, et al. Mucormycosis in patients with haematological malignancies: a retrospective clinical study of 37 cases. Br J Haematol 1997; 99:331-6.

4 Singh N, Gayowski T, Singh J, et al. Invasive gastrointestinal zygomycosis in a liver transplant recipient: case report and review of zygomycosis in solidorgan transplant recipients. Clin Infect Dis 1995;20:617-20.

5 Prabhu RM, Patel R. Mucormycosis and entomophthoramycosis: a review of the clinical manifestations, diagnosis and treatment. Clin Microbiol Infect 2004; 10(suppl 1):31-47.

6 Lee JH, Ha HK, Yoo E, et al. CT and sonographically guided biopsy in a patient with intestinal mucormycosis. AJR Am J Roentgenol 2000;175:129-31.

7 Nosari A, Oreste P, Montillo M, et al. Mucormycosis in hematologic malignancies: an emerging fungal infection. Haematologica 2000;85:1068-71.

8 Siu KL, Lee WH. A rare cause of intestinal perforation in an extreme low birth infant-gastrointestinal mucormycosis: a case report. J Perinatol 2004;24:319-21.

9 Ter Borg F, Kuijper EJ, Van der Lelie H. Fatal mucormycosis presenting as an appendiceal mass with metastatic spread to the liver during chemotherapyinduced granulocytopenia. Scand J Infect Dis 1990;22:499-501.

10 Petrikkos G, Skiada A, Sambatakou H, et al. Mucormycosis: ten-year experience at tertiary-care center in Greece. Eur J Clin Microbiol Infect Dis 2003;22:753-6. 
11 Thomson SR, Bade PG, Taams M, et al. Gastrointestinal mucormycosis. Br J Surg 1991;78:952-4.

12 Gomez-Lopez A, Cuenca-Estrella M, Monzon A, et al. In vitro susceptibility of clinical isolates of zygomycota to amphotericin B, fulcytosine, itraconazole and voriconazole. J Antimicrob Chemother 2001;48:919-21.

13 Johonson LB, Kauffman CA. Voriconazole: a new triazole antifungal agent. Clin Infect Dis 2003;36:630-7.

14 Cornely OA, Schmitzk K, Aisenbrey S. The first echinocandin: Caspofungin. Mycoses 2002;45:56-60.
15 Sun QN, Fothergill AW, McCarthy DI, et al. In vitro activities of posaconazole, itraconazole, voriconazole, amphotericin B and fluconazole against 37 clinical isolates of zygomycetes. Antimicrob Agents Chemother 2002;46:1581-2.

16 Sun QN, Najvar LK, Bocanegra R, et al. In vivo activity of posconazole against mucor species in an immunosuppressed-mouse model. Antimicrob Agents Chemother 2002;46:2310-12

17 Tedder M, Spratt JA, Anstadt MP, et al. Pulmonary mucormycosis: results of medical and surgical therapy. Ann Thorac Surg 1994;57:1044-50. 